\title{
HOMOTOPY EPIMORPHISMS AND LUSTERNIK-SCHNIRELMANN CATEGORY
}

\author{
JAMES A. DRAPER
}

\begin{abstract}
This paper examines the relationship of the LusternikSchnirelmann category and related numerical homotopy invariants to the epimorphisms in the homotopy category. The results are of the form: if $N$ is a numerical homotopy invariant and $f: X \rightarrow Y$ is an epimorphism, then under certain hypotheses $N(X) \geq N(Y)$. The Eckmann-Hilton dual of the main result is also included; as a corollary, a criterion is given for a categorical subobject of an $H$-space to be an $H$-space.
\end{abstract}

Introduction. The main result of this paper (Theorem 1.2) is that under certain dimension and connectivity restrictions on an epimorphism $f: X \rightarrow Y$ in the homotopy category, the LS-category of $Y$ does not exceed the LScategory of $X$; recall [12] $f$ is epic if $g f \simeq b f$ implies $g \simeq h$. This result is a generalization of the basic lemma in the theory of LS-category [6] which is that if $X$ dominates $Y$, then cat $Y$ does not exceed cat $X$. (In particular, this lemma implies that LS-category is a homotopy invariant.)

This result can be applied either to the problem of computing LScategory or to the problem of characterizing homotopy epimorphisms. At present both problems seem a long way from satisfactory solution, but such solutions should play a role in any successful structure theory for the homotopy category.

The first section of the paper contains the statement and proof of the main theorem. A corollary gives conditions under which a categorical quotient of a co-H-space is a co-H-space.

The second section describes the behavior of the related homotopy invariants cup-length, conilpotency and weak category with respect to epimorphisms. For each of those invariants the analogue of the main theorem is true without dimension and connectivity restrictions.

Received by the editors April 1, 1974.

AMS (MOS) subject classifications (1970). Primary 55C30, 55D45, 55D50, 55D99; Secondary 55D30.

Key words and phrases. Homotopy epimorphism, homotopy monomorphism, Lusternik-Schnirelmann category, cocategory, conilpotency, weak category, cuplength. 
The third section treats the Eckmann-Hilton dual of the main theorem. As a corollary there is a criterion for a categorical subobject of an $H$-space to be an $H$-space.

In this paper spaces are assumed to have the homotopy type of connected $\mathrm{CW}$-complexes and the dimension of a space $X, \operatorname{dim} X$, is the infimum of the dimensions of all CW-complexes having the homotopy type of $X$. The connectivity of $X$, conn $X$, is $n$ if $\pi_{j}(X)=0$ for $j \leq n$ and $\pi_{n+1}(X) \neq 0$. The (normalized) LS-category of $X$, cat $X$, is the smallest integer for which cat $X+1$ open sets, each contractible in $X$, cover $X$ or $\infty$ if no such integer exists.

1. Epimorphisms and category. The main theorem is proved using a natural sequence of fibrations $p_{n}(X): G_{n}(X) \rightarrow X$ defined by $\mathrm{T}$. Ganea [8]; this sequence characterizes LS-category as the lemma [8, Proposition 2.2] states.

Lemma 1.1. cat $X \leq n$ if and only if the fibration $p_{n}(X)$ admits a section, that is a map $s: X \rightarrow G_{n}(X)$ such that $p_{n}(X) s \simeq 1$.

Theorem 1.2. If $f: X \rightarrow Y$ is an epimorphism and $\operatorname{dim} Y \leq 2(k+1)$. (conn $Y+1)-3$, then cat $Y \leq \max (k$, cat $X)$.

Proof. The naturality of the fibration $p_{k}$ gives $f p_{k}(X) \simeq p_{k}(Y) G_{k}(f)$. When $k \geq$ cat $X, p_{k}(X)$ admits a section and so is epic; since $f$ is epic, $p_{k}(Y)$ is also epic.

A theorem of Y. Nomura [14, Theorem 6.2], modified by the use of Spanier's homotopy excision theorem [16] which removes the connectivity hypothesis, implies that if $\operatorname{dim} Y \leq 2 \operatorname{conn} p_{k}(Y)-1$, the epimorphism $p_{k}(Y)$ admits a section and so cat $Y \leq k$ by 1.1 .

The connectivity of $p_{k}(Y)$ can be computed inductively using the fact that $F_{k}$, the fibre of $p_{k}(Y)$, is homotopy equivalent to $F_{k-1} * \Omega Y$, the join of the fibre of $p_{k-1}$ and the loop space of $Y$ [7], [17]. For any $V$ and $W$, $\operatorname{conn}(V * W)=\operatorname{conn} V+\operatorname{conn} W+2$ and $\operatorname{conn} \Omega V=\operatorname{conn} V-1$, therefore $\operatorname{conn} F_{1}=\operatorname{conn}(\Omega Y * \Omega Y)=2 \operatorname{conn} Y$. Assuming inductively that $\operatorname{conn} F_{k}=$ $(k+1)(\operatorname{conn} Y+1)-2$,

$$
\begin{aligned}
\operatorname{conn} F_{k+1} & =\operatorname{conn}\left(F_{k} * \Omega Y\right)=\operatorname{conn} F_{k}+\operatorname{conn} \Omega Y+2 \\
& =(k+1)(\operatorname{conn} Y+1)-2+(\operatorname{conn} Y-1)+2 \\
& =(k+2)(\operatorname{conn} Y+1)-2 .
\end{aligned}
$$

Thus conn $p_{k}(Y)=\operatorname{conn} F_{k}+1=(k+1)(\operatorname{conn} Y+1)-1$ and the proof is complete. 
A space $Y$ is a homotopy quotient of a space $X$ if there is an epimorphism from $X$ to $Y$; the following is a criterion for a quotient of a co- $H$-space to be a co-H-space.

Corollary 1.3. Suppose $f: X \rightarrow Y$ is epic and $X$ is a co-H-space, then $Y$ is a co-H-space whenever $\operatorname{dim} Y \leq 4 \operatorname{conn} Y+1$.

Theorem 1.2 may be compared to the classical result for simply-connected spaces due to Grossman [11], cat $Y \leq \operatorname{dim} Y /$ conn $Y+1$. This indicates the strength of the hypothesis which assumes the existence of the epimorphism $f: X \rightarrow Y$. An even better comparison may be made with the following result of Berstein and Hilton [3, Corollary 3.12r] which does not require $f: X \rightarrow Y$ to be epic. $\left(\operatorname{cdim} C_{f}\right.$ denotes the cohomological dimension of the mapping cone of $f_{.}$)

Proposition 1.4. If cat $X \geq 1$ and conn $Y \geq 1, \operatorname{cdim} C_{f} \leq(k+1) \cdot$ (conn $Y+1)$ implies cat $Y \leq \max (k$, cat $X)$.

2. Epimorphisms and cup-length, conilpotency, weak-category. There are several numerical homotopy invariants related to LS-category. Each of them behaves nicely with respect to epimorphisms; in fact, the analogues of Theorem 1.2 are proved for these invariants without any dimension or connectivity hypotheses.

The cup-length of a space $X$, cup-long $X$, is the length of the longest nontrivial cup product of positive-dimensional singular cohomology classes with any ring of coefficients. Clearly an analogous definition may be made for any multiplicative cohomology theory and the result below remains valid.

Proposition 2.1. If $f: X \rightarrow Y$ is epic, cup-long $X \geq$ cup-long $Y$.

Proof. By [15], the suspension of $f$ admits a section, therefore the suspension isomorphism theorem for cohomology implies $f^{*}$, the induced map in cohomology, has a left inverse and any nontrivial product in the cohomology of $Y$ corresponds to a nontrivial product in the cohomology of $X$.

Example 2.2. The Grassmann manifolds are not homotopy quotients of the Stiefel manifolds; in particular, the projective spaces are not homotopy quotients of the spheres. This is an immediate consequence of 2.1 and the known structure of the cohomology algebras of the Grassmann and Stiefel manifolds (see, e.g., [4, Chapter 8]).

The weak category of a space $X$, wcat $X$, was defined by Berstein and Hilton [2]. By definition, wcat $X \leq n-1$ if and only if the composite 
$q_{n}(X) d_{n}(X)$ is nullhomotopic, where $d_{n}(X): X \rightarrow X^{n}$ is the diagonal map and $q_{n}(X): X^{n} \rightarrow X^{(n)}$ is the canonical map to the reduced product.

Proposition 2.3. If $f: X \rightarrow Y$ is epic, wcat $X \geq$ wcat $Y$.

Proof. If wcat $X \leq n-1, q_{n}(X) d_{n}(X) \simeq 0$. By naturality

$$
q_{n}(Y) d_{n}(Y) f \simeq q_{n}(Y) f^{n} d_{n}(X) \simeq f^{(n)} q_{n}(X) d_{n}(X) \simeq 0
$$

Since $f$ is epic, $q_{n}(Y) d_{n}(Y) \simeq 0$ and wcat $Y \leq n-1$.

The conilpotency of a space $X$, conil $X$, is defined to be the maximal length of a nontrivial commutator in the cogroup $\Sigma X[1]$. Thus there are maps $\Psi_{n}(X): \Sigma X \rightarrow{ }^{n} \Sigma X$ (the $n$-fold sum of $\Sigma X$ with itself) such that conil $X \leq$ $n-1$ if and only if $\Psi_{n}(X) \simeq 0$.

Proposition 2.4. If $f: X \rightarrow Y$ is epic, conil $X \geq$ conil $Y$.

Proof. Suppose conil $X \leq n-1$, then $\Psi_{n}(X) \simeq 0$. By naturality $\Psi_{n}(Y) \Sigma f \simeq{ }^{n}(\Sigma f) \Psi_{n}(X) \simeq 0$. Since $f$ is epic, $\Sigma f$ has a section [15] and hence $\Psi_{n}^{n}(Y) \simeq 0$.

There are generalizations of 2.3 and 2.4 in terms of right homotopy structures in the sense of Peterson [3]; these are omitted to avoid excessive notation and terminology. For details see [5].

It is well known that for each space $X$,

$$
\text { cat } X \geq \text { wcat } X \geq \text { conil } X \geq \text { cup-long } X
$$

and that strict inequality is possible (see e.g. [10]). There are also invariants whose values are upper bounds for LS-category; for example by [8, Corollary 3.3] if conn $X \geq 1$, cat $X \leq \mathrm{h}-\operatorname{dim} X /$ conn $X+1=T(X)$, where $\mathrm{h} \operatorname{dim} X$ denotes the homological dimension of $X$. Clearly if $f: X \rightarrow Y$ is epic, $T(X) \geq T(Y)$. These facts make the necessity of the dimension and connectivity hypotheses of Theorem 1.2 an intriguing open question.

3. Monomorphisms and cocategory. The dual of an epimorphism is a monomorphism [12], i.e. a map $f$ such that whenever $f g \simeq f h, g \simeq h$. The classical example of a monomorphism is the Hopf map $p: s^{3} \rightarrow s^{2}$ (see e.g. [13, p. 69]).

There is a natural sequence of cofibrations $e_{k}(X): X \rightarrow T_{k}(X), k \geq 0$, defined by T. Ganea [7]. The following definition is the dual of 1.1 .

Definition 3.1. The cocategory of $X$ does not exceed $n$, cocat $X \leq n$, if $e_{n}(X)$ admits a left homotopy inverse; cocat $X=n$ if cocat $X \leq n$ but it is false that cocat $X \leq n-1$, and cocat $X=\infty$ otherwise. 
The following theorem is the Eckmann-Hilton dual of Theorem 1.2. Since the proof is the dual of the proof of 1.2 only a sketch is provided.

Theorem 3.2. If $f: X \rightarrow Y$ is monic and $\pi_{i}(X)=0$ for $i \geq$ $2(k+1) \operatorname{conn} X+3$, then cocat $X \leq \max ($ cocat $Y, k)$.

Proof. If $k \geq \operatorname{cocat} Y, e_{k}(X)$ is monic. By induction on the formula in [9, Theorem 2.1], conn $e_{k}(X)=(k+1) \operatorname{conn} X+1$. By [14, Theorem 4.1] if $e_{k}(X)$ is monic and if $\pi_{i}(X)=0$ for $i \geq 2$ conn $e_{k}(X)+1, e_{k}(X)$ admits a left homotopy inverse and cocat $X \leq k$.

It is well known that a space $X$ is an $H$-space if and only if cocat $X \leq 1$. If there is a monomorphism $f: X \rightarrow Y, X$ is a homotopy subobject of $Y$. Thus 3.2 implies the following result.

Corollary 3.3. If $X$ is a homotopy subobject of an $H$-space $Y$ and $\pi_{i}(X)=$ 0 for $i \geq 4 \operatorname{conn} X+3, X$ is an $H$-space.

There are also questions about the relationships between monomorphisms and LS-category and between epimorphisms and cocategory. Note that the basic lemma of LS-category can also be interpreted as stating that if $g: Y \rightarrow$ $X$ is a monomorphism with a left homotopy inverse $f: X \rightarrow Y$, then cat $Y \leq$ cat $X$. Results in this direction have not been too promising; however, there are the following corollaries to Nomura's results [14, Theorems 4.1 and 6.2] which imply that under the given hypotheses $X$ dominates $Y$.

Proposition 3.4. If $g: Y \rightarrow X$ is an n-connected monomorphism $(n \geq 1)$ and $\pi_{i}(Y)=0$ for $i \geq 2 n+1$, then cat $Y \leq$ cat $X$. If $f: X \rightarrow Y$ is an $n$-connected epimorphism $(n \geq 1)$ and $\operatorname{dim} Y \leq 2 n-1$, cocat $Y \leq$ cocat $X$.

\section{REFERENCES}

1. I. Berstein and T. Ganea, Homotopical nilpotency, Illinois J. Math. 5 (1961), 99-130. MR 23 \#A3573.

2. I. Berstein and P. J. Hilton, Category and generalized Hopf invariants, Illinois J. Math. 4 (1960), 437-451. MR 23 \#A3572.

3. - Homomorphisms of homotcpy structures, Topol. et Géom. Diff. (Sém. C. Ehresmann), Vol. 5, Institut H. Poincare, Paris, 1963, 24 pp. MR 34 \#3574.

4. A. Dold, Lectures on algebraic topology, Springer-Verlag, New York, 1972.

5. J. Draper, Epimorphisms in the homotopy cate gory, Dissertation, University of Washington, 1972.

6. R. H. Fox, On the Lusternik-Schnirelmann category, Ann. of Math. (2) 42 (1941), 333-370. MR 2, 320.

7. T. Ganea, A generalization of the homology and homotopy suspension, Comment. Math. Helv. 39 (1965), 295-322. MR 31 \#4033.

8. - Lusternik-Schnirelmann category and strong category, Illinois $\mathrm{J}$. Math. 11 (1967), 417-427. MR 37 \#814. 
9. T. Ganea, On the homotopy suspension, Comment. Math. Helv 43 (1968), 225-234. MR 37 \#4813.

10. W. J. Gilbert, Some examples for weak category and conilpotency, Illinois J. Math. 12 (1968), 421-432.

11. D. P. Grossman, An estimation of the category of Lusternik-Schnirelmann, C. R. (Doklady) Akad. Sci. URSS (N. S.) 54 (1946), 109-112. MR 8, 334.

12. P. J. Hilton, Homotopy theory and duality, Gordon and Breach, New York, 1965. MR $33 \# 6624$.

13. S. T. Hu, Homotopy theory, Pure and Appl. Math., vol. 8, Academic Press, New York, 1959. MR 21 \#5186.

14. Y. Nomura, The Whitney join and its dual, Osaka J. Math. 7 (1970), 353373. MR $43 \# 6927$.

15. D. Puppe, Homotopiemengen und ihre induzierten Abbildungen. I, Math. Z. 69 (1958), 299-344. MR 20 \#6698.

16. E. Spanier, The homotopy excision theorem, Michigan Math. J. 14 (1967), 245-255. MR 34 \#6771.

17. J. Rutter, On a theorem of T. Ganea, J. London Math. Soc. (2) 3 (1971), 190-192. MR 43 \#5532.

DEPARTMENT OF MATHEMATICS, UNIVERSITY OF FLORIDA, GAINESVILLE, FLORIDA 32611 